\title{
Power Flow Optimization Problems for Multi-Energy Systems
}

\author{
Dmitry Bykov ${ }^{1, *}$, and Dmitry Efimov ${ }^{1,2}$ \\ ${ }^{1}$ Melentiev Energy Systems Institute, Electric Power System Department, 664033 Irkutsk, Russia \\ ${ }^{2}$ Irkutsk National Research Technical University, Energy Institute, 664074 Irkutsk, Russia
}

\begin{abstract}
Flow distribution calculation is the determination of the values of the state parameters at the nodes and connections of the system that satisfy the Kirchhoff laws. This calculation is necessary to determine the existence and admissibility of operating states in a multi-energy systems, as well as in singleproduct systems. In addition, it is an essential component of solving the problems of optimization of states in order to ensure the minimum cost of production, transmission and distribution of energy resources. Traditionally, a means of such minimization is the redistribution of the load between sources, consumers and storage of energy resources. As a consequence, this redistribution applies to the elements of transmission and distribution networks. This means are supplemented also by the possibility of converting energy resources from one type to another in multi-energy system. Covering the needs of the end consumer in different types of energy can be provided from different primary energy carriers through the chain (sequence) of their transfer and transformation from one type to another. Such a variety of the ways of energy supply, along with the possibilities of storing (accumulating) energy, pro-vides necessary degrees of freedom for solving states optimization problems.
\end{abstract}

\section{Allowable and optimal power flow conditions}

The calculation of the allowed conditions consists in determining the values of dependent and independent parameters. These parameters must satisfy the following conditions: the equations of the flow distribution model are fulfilled, and the controlled values (dependent and independent parameters of the state, as well as functions from them) correspond to technical constraints in the form of equalities and inequalities. To be able to comply with the restrictions, the number of dependent parameters must exceed the number of flow distribution equations per number of restrictions [1].

State optimization problems are solved in conditions of operational and automatic states control of power systems and grids. The calculation of the optimal state consists in determining such an allowed state which will have the objective function is equal to the minimum (or maximum) value. In this case, the flow distribution equations act as equality constraints on the state parameters. Costs for the time interval between two control actions, or costs per unit of time for a given consumer load at each moment of time are used as the target function. Variable cost components, which depends on the network conditions, are often taken as the target (minimized) function.

When optimizing the current state of the power system according to the power generation of energy resources, the volumes distribution of the produced resource per unit of time is determined. In this case, the producers' capacity injections that correspond to the min- imum operating costs for given consumer loads are determined. A more particular problem is to optimize the electrical grid conditions. This means the calculation of the optimal node voltage, reactive power of sources and transformation ratios of variable transformers and autotransformers, taking into account technical constraints to minimize losses [2]. The problems of optimizing the power system conditions and the grid conditions can be solved jointly (the so-called complex optimization of the operating conditions) [3]. However, separate optimization is used more often to simplify the task [4]: the power system conditions is optimized first, and then the power grid conditions is optimized.

\section{Operating cost minimization}

One of the obvious ways to improve production efficiency is to reduce production costs with no structural changes in the system. Operating costs reduction, environmental impact, energy efficiency is taken into account as the criteria. Various optimization strategies are used - following electrical load, heat load and hybrid [5].

\subsection{Gas and power system}

In [6], the problem is to minimize the total operating costs in gas-electric system. At the same time, it is necessary to satisfy the demand requirements over the entire time horizon, which is set at 1 month. The time step is chosen as 1 day. The optimizations function as follows: 


$$
\min \sum_{t}^{\text {Horizon }}\left(\begin{array}{c}
\sum_{a=1}^{N Q} \mu C_{t} Q_{a, t}^{\text {Supp }}+\sum_{k=1}^{N L} \mu C_{t} Q_{k, t} \\
+\sum_{s=1}^{N S} \mu\left(-Q_{s, t} I C_{s, t}+Q_{s, t} W C_{s, t}\right) \\
+\sum_{b=1}^{N G G} \mu C_{b} Q_{b, t}^{\text {GasShed }}+\sum_{i=1}^{N G} \lambda C_{i} P_{i, t}^{G e n} \\
+\sum_{j=1}^{N G E} \lambda C_{j} P_{j, t}^{\text {Elecshed }}
\end{array}\right),
$$

where

$C_{t, b, i, j}$ are costs of gas, gas load shedding, power generator, electricity load shedding respectively;

$Q$ is gas flow;

$P$ is power output at a time;

$\lambda, \mu$ are electrical and gas units per day conversion coefficients (24 and 86400).

The authors of [7] propose to use the multi-agent genetic algorithm (MAGA) to solve the problems of multi-carrier systems optimal power flow (MCOPF) in general, as well as optimal flow distribution of electrical (OPF) and gas (GOPF) systems in particular.

A formulation of the optimal flow distribution method for the gas network (GOPF) is obtained based on the equations for gas systems (flow equation, flow balance equation):

$$
\min \sum_{i=1}^{N} G p r_{i} \omega_{s i}
$$

where

$G p r_{i}$ is gas price at node $i$;

$\omega_{s i}$ is gas injection.

The authors propose to use the energy hub as a place of concentration of several energy carriers for the MCOPF solution. The general formulation of the method for calculating in a hub is:

$$
\min f(\boldsymbol{P}, \boldsymbol{F}, \boldsymbol{v}),
$$

where

$\boldsymbol{P}$ is energy hub power inputs vector;

$\boldsymbol{F}$ is vector of different network flows;

$\mathrm{V}$ is dispatch factor of energy hub;

\subsection{Heat and power system}

The authors of [8] propose an IEMS that will predict electrical and thermal loads to optimize microgrid costs. The system includes an intelligent storage system consisting of electric (chemical batteries) and thermal (internal ice melting technology) storages.

The aim of optimization is to simultaneously satisfy electrical and thermal loads at the lowest cost. Operating costs include the costs of power transmission, maintenance, costs of turning the system on and off, exchanging power with utility and purchasing gas for the booster heater. The cost function is:

$$
\begin{aligned}
& \min \sum_{t=1}^{T}\left\{\sum _ { i = 1 } ^ { N _ { g } } \left[u _ { i } ( t ) P _ { g i } ( t ) \left(B_{g i}(t)\right.\right.\right. \\
& \left.\left.+K_{\text {OMi }}\right)+S_{g i}\left|u_{i}(t)-u_{i}(t-1)\right|\right] \\
& +\sum_{j=1}^{N_{E S}}\left[u_{j}(t) P_{S j}(t) B_{S j}(t)\right] \\
& +P_{\text {Grid }}(t) B_{\text {Grid }}(t) \\
& \left.+\left(C_{\text {gas }}+K_{\text {OMbl }}\right) G_{b l}(t)\right\}
\end{aligned}
$$

where

$u_{i}(t), u_{j}(t)$ are conditions of units $i, j$ at hour $t$;

$P_{g i}(t)$ is active power generation of $i$-th DG unit (Distributed Generation);

$P_{S j}(t)$ is charge/discharge power of the $j$-th storage athour $\mathrm{t}$;

$P_{\text {Grid }}(t)$ is active power which is bought (sold) from (to) the utility at hour $t$;

$B_{g i}(t), B_{S j}(t), B_{\text {Grid }}(t)$ are the bids of $i$-th DG unit, $j$-th storage device and utility at hour $t$;

$K_{O M i}, K_{O M b l}$ are the coefficients which depend on maintenance cost of generator unit $i$ and auxiliary boiler, respectively;

$N_{g}, N_{E s}$ are total numbers of generators and batteries;

$S_{g i}$ is the start-up or shut-down cost of $i$-th DG unit;

$C_{g a s}$ is the price of natural gas;

$G_{b l}(t)$ is consumption of natural gas for auxiliary boiler at time $t$.

In addition there is the function of minimization of air emissions:

$$
\begin{aligned}
\min \sum_{t=1}^{T}\{( & \left.\sum_{i=1}^{T_{E}} \sum_{j=1}^{N} E F_{i j} P_{g i}(t)\right) \\
& +P_{b l}(t) E F_{b l} \\
& \left.+P_{\text {Grid }}(t) E F_{\text {grid }}\right\},
\end{aligned}
$$

where

$E F_{i j}, E F_{b l}, E F_{\text {grid }}$ are emission factors of $i$-th generator unit, emission type $j$, for the utility and the auxiliary boiler, respectively;

$P_{b l}(t)$ is output thermal energy of auxiliary boiler.

\subsection{Gas, heat and power system}

A model that calculates the amount of required energy carrier based on the current load and the cost of energy is presented in [9]. The optimization criteria is the cost of the energy carrier in the selected period of time.

The authors model the hub for research at first, using the following assumptions:

- the system is in steady state;

- energy losses within the hub occur only on converters and storage devices.

Much of time periods are examined, and decisions on the energy produced are made for each period separately to define the optimal control of the energy hub. The task 
of planning the operation of an energy hub includes the equations of energy storage devices, storage capacity limits, power and energy limits. The optimization problem in the initial conditions looks as follows:

$$
\min \sum_{k=0}^{T-1} c(k)^{\prime} \mathbf{P}(k) \text {, }
$$

where

$c(k)^{\prime}$ is energy purchasing costs for each energy input varying at each point in time $k$;

$P(k)$ is the power input vector at time $k$.

The authors propose to solve the classical problem of reliable control simultaneously with the planning problem for a robust hub. The restrictions and conditions set by this problem will be discussed later. Accordingly, the task of planning the operation of a robust energy hub will take the form:

$$
\min \sum_{k=0}^{T-1} c(k)^{\prime} \mathbf{P}(k)+\rho\|\Sigma(k)\|_{2}^{2},
$$

where

\section{$\rho$ is a penalty weight;}

$\Sigma(k)$ is a vector of auxiliary variables introduced to account for equality constraints violations.

\subsection{Hierarchical approach to optimization}

As the target (minimized) function for short-term steadystate mode of a multi-energy system there is the total operating costs for the production, transmission and distribution of energy resources per unit of time [10]. The function is as follows:

$$
\min \left[Z^{1}+\sum_{i=1}^{N_{2}}\left(Z_{i}^{2}+\sum_{j=1}^{N_{3}} Z_{j, i}^{3}\right)\right],
$$

where

$Z^{1}$ are energy costs in the first level system;

$Z_{i}^{2}$ are energy costs in the $i$-th system at second level;

$Z_{j, i}^{3}$ are energy costs in the $j$-th system at third level, connected with $i$-th system at second level;

$N_{2}$ is the amount of systems at second level;

$N_{3}$ is the amount of systems at third level.

\section{Power optimization}

Another way to improve efficiency is to increase the turnover of energy resources, provided there is no replacement of system elements. Optimization can be carried out through the power consumed by the system or the power flow distribution of the system.

\subsection{Power losses minimization}

In [11], the authors propose methods of voltage gradation and network separation for solving the problem of optimizing large power systems. Optimization is based on the problem of nonlinear non-convex programming. The solution is achieved by optimizing the objective function using optimization algorithms that satisfy the equality constraints (power flow equation) and inequality constraints (safety constraints). The objective function is:

$$
\min \left(\sum_{i \in S_{G}} P_{G i}-\sum_{i \in S_{D}} P_{D i}\right),
$$

where $P_{G i, D i}$ is the active power of generator and active power of load in node $i$ respectively.

According to the authors, the application of the methods of gradation and separation is possible in the conditions of a radial network in an open loop mode. In this case, the network is divided into several relatively independent subnets of different voltage levels, and each subnetwork is connected to the main high voltage network through border nodes. The optimization function after grading voltage levels and grading the network looks as follows:

$$
\min \sum_{i=0}^{N} f_{i}\left(x_{i}\right)
$$

where $x_{i}$ are the control variables and state variables of the subnet $i$.

According to the authors, the model they created on the basis of the described method "significantly reduces the dimension of the matrix of coefficients of the correction equation."

\subsection{Production maximization}

The optimization problem in [12] is to maximize the generation of electricity and recovered heat. The authors have modeled a loopback system consisting of electrical and hydraulic networks with several elements of distributed generation, which are thermal power plants. It is also accepted that the generation of electricity depends on the electrical model of the system, heat - on the electrical, water and heat-and-power models of the system. The function of the generators output power was selected for optimization:

$$
\max \sum_{g \in G} \sum_{n \in N}\left(P_{g, n}^{F E}+P_{g, n}^{F T}\right),
$$

where

$G$ is set of new generators;

$N$ is set of electrical buses

$P_{g, n}^{F E}$ is final electrical power output of $g$ at $n$

$P_{g, n}^{F T}$ is final thermal power output of $g$ at $n$.

\section{Control parameters constraints}

An important element of the optimization problem formulation is setting constraints on the objective function.

One of the main constraints applied to the optimization function in the power system is the limitation of the minimum and maximum values of the parameters of the system elements. These limits are process and / or network bandwidth dependent. Various parameters of the optimized system (power, voltage, pressure, capacity, flow distribution, etc.) can act as 
constraints. Constraints can be represented in vector form $[7,11]$, but scalar form is used more often [8, 9, 12]. These constraints are inequality constraints. Inequality constraints can be two-sided and one-sided. Equality constraints are specified for the optimization function in addition to inequality constraints. Equations of power balance, flow distribution, etc. act as such constraints. In addition, in some sources the constraints used as the connection between energy resources or energy flows of different nature. This is only applies to multi-energy systems [6].

The more restrictions are specified, the more accurately the function will be defined. In all sources, the authors model each element of the studied system that can affect the result, be it large system generating plants $[7,12]$, nodes of distribution systems $[5,11]$ or even household energy consumers (refrigerators, air conditioners, water heaters, etc.) [9].

\subsection{Electrical power system}

The optimization function using the voltage gradation and network separation method is presented in subsection 3.1, equation (9). This function has the following constraints [11]:

$$
\begin{gathered}
P_{G i}-P_{D i}-e_{i} \sum_{j=1}^{n}\left(G_{i j}-B_{i j} f_{j}\right) \\
-f_{j} \sum_{j=1}^{n}\left(G_{i j}+B_{i j} e_{j}\right)=0, \\
Q_{G i}-Q_{D i}-f_{i} \sum_{j=1}^{n}\left(G_{i j} e_{j}-B_{i j} f_{j}\right) \\
+e_{j} \sum_{j=1}^{n}\left(G_{i j} f_{j}+B_{i j} e_{j}\right)=0, \\
\underline{P}_{G i} \leq P_{G i} \leq \bar{P}_{G i}, \quad i \in S_{G}, \\
\underline{Q}_{R i} \leq Q_{R i} \leq \bar{Q}_{R i}, \quad i \in S_{R}, \\
\underline{V}_{i} \leq v_{i} \leq \bar{V}_{i}, \quad i=1,2,3 \ldots n, \\
\underline{P}_{i j} \leq P_{i j} \leq \bar{P}_{i j}, \quad i, j \in S_{L},
\end{gathered}
$$

where

$Q_{R i}$ is the reactive power compensation capacity of node

$i$;

$V_{i}$ is the voltage amplitude of node $i$;

$P_{i j}$ is the active power of transmission line $i j$;

$e_{i}, f_{i}$ are the real part and imaginary part of the voltage at bus $i$ respectively;

$S_{G}, S_{R}, S_{L}$ are the set of thermal plants, reactive power source and constrained lines, respectively.

The general formulation of constraints is also described for the method:

$$
\left\{\begin{array}{c}
m_{i}\left(x_{1}, x_{2}, \ldots x_{n}\right)=0 \\
h_{i}\left(x_{i}\right)=0 \\
\underline{g_{i}}\left(x_{i}\right) \leq g_{i}\left(x_{i}\right) \leq \overline{g_{i}}\left(x_{i}\right)
\end{array},\right.
$$

where $x_{i}$ are the control variables and state variables of the subnet $i$;

$h_{i}\left(x_{i}\right)$ is equality constraints;

$m_{i}\left(x_{1}, x_{2}, \ldots x_{n}\right)$ is the boundary coupling constraint condition among the subnets;

$g_{i}\left(x_{i}\right)$ is inequality constraint.

By introducing the slack variable vector $l_{i}, u_{i}$ for each subnet, inequality constraints converted into the equation constraints. Equation (15) is transformed to:

$$
\left\{\begin{array}{c}
m_{i}\left(x_{1}, x_{2}, \ldots x_{n}\right)=0 \\
h_{i}\left(x_{i}\right)=0 \\
g_{i}\left(x_{i}\right)-l_{i}-g_{i}\left(x_{i}\right)=0 \\
g_{i}\left(x_{i}\right)+u_{i}-\overline{g_{i}}\left(x_{i}\right)=0 \\
l_{i} \geq 0, u_{i} \geq 0
\end{array} .\right.
$$

For the system consisting of equalities, the improved approximate Newtonian direction method is used, and then the nonlinear primal dual interior point method is applied to the resulting system.

\subsection{Gas and power system}

or the optimization function for a gas-electric system (equation 1), all constraints can be divided according to the type of energy resource [6]:

- For gas:

$$
\begin{aligned}
M_{u} Q^{\text {supp }}+M_{p} Q_{n} & +M_{c} Q_{n}^{c}-M_{t} \tau^{c} \\
& +M_{s} Q_{s} \\
& =M_{d}\left(Q^{\text {Gdem }}\right. \\
& \left.-Q^{\text {GShed }}\right) \\
p^{\text {min }} \leq p & \leq p^{\text {max }},
\end{aligned}
$$

where

$M$ is node incidence matrix;

$Q$ is flowrate;

$p$ is pressure;

- For electricity.

$$
\begin{gathered}
\sum_{i}^{G} P_{i, t}^{\text {Gen }}=\sum_{j}^{D d} P_{j, t}^{\text {Dem }}-\sum_{j}^{D d} P_{j, t}^{\text {Elecshed }}, \\
P_{i}^{\text {Gen(min) }} \leq P_{i}^{\text {Gen }} \leq P_{i}^{\text {Gen }(\max )},
\end{gathered}
$$

where $P^{G e n}$ is power output of generation unit $i$ at time $t$;

- The relationship between the gas fuel flow and the real electrical power generated is expressed as:

$$
P^{G e n}=\varphi Q_{n} H_{g},
$$

where

$\varphi$ is thermal efficiency of the gas turbine;

$Q_{n}$ is flowrate through the compressor at standard conditions;

$H_{g}$ is gas heating value $\left(\sim 39 \mathrm{MJ} / \mathrm{m}^{3}\right)$.

\subsection{Heat and power system}

In [12], the authors carried out work on modeling electrical, plumbing and hydraulic systems. The following elements were included in the model - pump 
equation, water head drop, nodal balance equations, nodal head limitation, water temperature limitation, turbine water consumption limitation, ambient temperature limitation and recovered heat output equation. The constraints of the function (equation 11) for the electrical system are presented below:

$$
\begin{gathered}
P_{g, n}^{(E, T)-} \leq p_{g, n}^{(E, T)} \leq P_{g, n}^{(E, T)+}, \forall g \in G \forall n \in N \\
V_{n}^{-} \leq v_{n} \leq V_{n}^{+}, \\
C_{c, n}^{-} \leq s_{c, n}^{C} \leq C_{c, n}^{+}, \\
\sqrt{\left(f_{l}^{(A, B) P}\right)^{2}+\left(f_{l}^{(A, B) Q}\right)^{2}}=\left(F_{l}^{+}\right)^{2}, \forall l \in L .
\end{gathered}
$$

\subsection{Gas, heat and power system}

Restrictions below apply to the optimization function (equation 2) from paragraph 2.1. They are all two-sided:

$$
\begin{gathered}
1 \leq \frac{P_{k}}{P_{m}} \leq R_{j \max }, \quad j=1, \ldots, N_{C}, \\
P_{i \min } \leq P_{i} \leq P_{i \max }, \quad i=1, \ldots, N_{n}, \\
\omega_{\text {s min }} \leq \omega_{s i} \leq \omega_{s \max }, i=1, \ldots, N_{S},
\end{gathered}
$$

where

$P_{i}$ is pressure at node $i$;

$R_{\text {j max }}$ is max compression ratio at node $j$;

$P_{k}, P_{m}$ are suction and discharge pressures;

$N_{C}, N_{n}, N_{S}$ are numbers of compressors, modes and supplies in gas network, respectively.

\section{Conclusion}

The system balance equations, power balance equations, element parameters, etc. act as constraints on the mode parameters in optimization problems. It is possible to trace the continuity of the formulations and methods for solving problems of optimization of the regime of a multi-energy system with similar optimization problems for single-product systems.

When setting the optimization problem, it is necessary to choose an objective function. The most common are cost optimization and capacity optimization. As can be seen from the sections, cost optimization is more popular among authors, since increasing profitability is the primary goal of any large-scale production.

Constraints are an integral part of any optimization task. It is the constraints that define the boundaries within which the objective function will be defined. Therefore, the correct selection of equality constraints and inequality constraints is necessary to obtain a correct and accurate solution.

The research was carried out under State Assignment Project (no. FWEU-2021-0001, registration no. AAAAA21-121012190027-4) of the Fundamental Research Program of Russian Federation 2021-2030.

\section{References}

1. D.N. Efimov, D.M. Bykov «Load-flow model of a multi-energy system» // E3S Web of Conferences, 139 (2019).

2. V.I. Idel'chik «Elektricheskie sistemy i seti: Uchebnik dlya vuzov» - M.: Energoatomizdat, (1989).

3. V.E. Vorotnickij, YU.S. ZHelezko, V.N. Kazancev «Poteri elektroenergii V elektricheskih setyah energosistem»- M.: Energoatomizdat (1983).

4. G.E. Pospelov, N.M. Sych «Poteri moshchnosti i energii v elektricheskih setyah»-M.: Energoizdat, (1981).

5. P.J. Mago, L.M. Chamra «Analysis and optimization of CCHP systems based on energy, economical, and environmental considerations» Energy and Buildings, 41 (2009).

6. M. Chaudry, N. Jenkins, G. Strbac «Multi-time period combined gas and electricity network optimization» Electric Power Systems Research 78 (2008).

7. M. Moeini-Aghtaie, A. Abbaspour, M. FotuhiFiruzabad, E. Hajipour, «A Decomposed Solution to Multiple-Energy Carriers Optimal Power Flow» IEEE Trans on Power Systems 29 (2014).

8. M. Motevasel, A. R. Seifi, T. Niknam. «Multiobjective energy management of CHP-based microgrid» Energy 51 (2013).

9. A. Parisio, C. Del Vecchio, G. Velotto «Robust optimization of operations in energy hub» 50th IEEE Conference on Decision and Control and European Control Conference, Orlando, FL, (2011).

10. E.A. Barahtenko, O.N. Vojtov, N.I. Voropaj, V.A. Stennikov «Ierarhicheskoe modelirovanie intellektual'nyh integrirovannyh energeticheskih sistem» Ierarhicheskoe modelirovanie sistem energetiki Izd-vo «Geo» (2020)

11. Y. Yude, W. Zhongbiao, Z. Yiyi, W. Hua, «Largescale OPF Based on Voltage Grading and Network Partition» CSEE Journal of power and energy systems 2 (2016).

12. X. Zhang, G.G. Karady, K.R. Piratla, S.T. Ariaratnam «Network Capacity Assessment of Combined Heat and Power-Based Distributed Generation in Urban Energy Infrastructures» // IEEE Trans. on Smart Grid 4 (2013). 